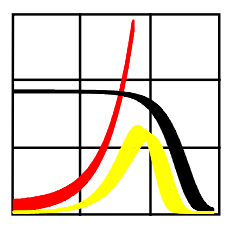

Max-Planck-Institut für demografische Forschung

Max Planck Institute for Demographic Research

Doberaner Strasse 114 - D-18057 Rostock · GERMANY

Tel +49 (0) 3812081 - 0; Fax +49 (0) 3812081 - 202;

http://www.demogr.mpg.de

Cancer Rates over Age, Time, and

Place: Insights from Stochastic

Models of Heterogeneous

Populations

James W. Vaupel (jwv@demogr.mpg.de)

Anatoli I. Yashin (yashin@demogr.mpg.de)

C Copyright is held by the authors.

Working papers of the Max Planck Institute for Demographic Research receive only limited review. Views or opinions expressed in working papers are attributable to the author(s) and do not necessarily reflect those of the Institute. 


\title{
Cancer Rates over Age, Time, and Place: Insights from Stochastic Models of Heterogeneous Populations
}

\author{
James W. Vaupel and Anatoli I. Yashin \\ Max Planck Institute for Demographic Research \\ Rostock, Germany
}

Total word count $=10,721$

Text word count $=9,135$

This research was originally reported in January 1988 in Working Paper \#88-01-1 of the Center for Population Analysis and Policy, University of Minnesota. It has not been revised or updated.

\begin{abstract}
Individuals at the same age in the same population differ along numerous risk factors that affect their chances of various causes of death. The frail and susceptible tend to die first. This differential selection may partially account for some of the puzzles in cancer epidemiology, including the lack of apparent progress in reducing cancer incidence and mortality rates over time.
\end{abstract}

\section{Introduction}

The epidemiology of cancer seems peculiar, puzzling, even paradoxical:

- cancer incidence and mortality rates level off or even decline at older ages;

- cancer accounts for a greater proportion of deaths around ages 50 or 60 than at younger or older ages;

- when the age trajectories of cancer rates for two cohorts, from different time periods, countries, histological types, etc., are compared, a crossover or at least some convergence with age is frequently evident, so that the cohort that is disadvantaged at younger ages is less disadvantaged or even advantaged at older ages;

- incidence and mortality rates from various forms of cancer differ far more from country to country than do rates of total cancer incidence and mortality;

- countries with high age-specific cancer rates tend to have low age-specific mortality rates from other causes and countries with high age-specific mortality rates from other causes tend to have low age-specific cancer rates;

- mortality from some forms of cancer, notably lung cancer, is increasing, whereas mortality from some other forms of cancer, especially stomach cancer and cancer of the uterus, is decreasing;

- overall, cancer incidence and mortality rates are tending to somewhat increase, whereas mortality rates from almost all other major causes of death, including heart disease and cerebrovascular disease, are declining.

The various forms of cancer and other causes of death can be considered competing risks. In analyzing patterns of cause-specific mortality, two assumptions are usually made: 
(1) individuals of the same age in the same population group face the same probability of death from each of the various causes, and

(2) the competing risks are independent of each other: a change in the force of mortality (i.e., the intensity or hazard of death by age) from any specific cause is assumed to have no impact on the force of mortality from any other cause [8, 9, 24, 61].

The first assumption is clearly inconsistent with the generally recognized fact that individuals, even of the same age and in the same population or subpopulation, differ in their overall frailty and in their susceptibility to various causes of death. Physicians, epidemiologists, biostatisticians, and demographers have long recognized that individuals differ along numerous risk factors, some observed and others unobserved, some genetic in origin and others resulting from behavior and environment exposure, that influence morbidity and mortality rates.

We show that this heterogeneity implies that the second assumption is also invalid: in a heterogeneous population competing risks of death will not, except in special circumstances, be independent on the population level, even though the competing risks may be independent for every individual in the population.

Even if an assumption is invalid it might be useful as a simplifying approximation. The question thus is: are the effects of heterogeneity and the resulting dependency among competing risks small enough to be ignored? The models we develop suggest that the effects might be sufficiently substantial to at least partially account for the puzzles and paradoxes in the epidemiology of cancer listed above.

That selection in a heterogeneous population might be at least a contributing cause of the leveling off and decline with age in cancer rates is a hypothesis familiar to cancer epidemiologists, although it has tended to be dismissed. Therefore, we start with this effect and devote a large share of the article to scrutinizing it.

Two major conclusions emerge:

(1) a variety of alternative models of selection and competing risks can produce the leveling off and decline, and even if most of these models are implausible, some variant or other may prove appropriate, and

(2) even if substantial heterogeneity exists in a population no noticeable leveling off or decline may be apparent, so the absence of this kind of effect is not evidence against heterogeneity.

We then turn to illustrating how heterogeneity may be at least a partial cause of puzzles and paradoxes of cancer epidemiology involving variation in cancer rates over time and place, instead of over age. Probably our most significant finding, given the scientific and policy interest in cancer trends, is that observed cancer rates in a heterogeneous population may increase over time even though the risks of cancer for individuals are declining. We explain three different mechanisms that can produce this effect.

\section{Age Patterns}

\subsection{The Rise and Fall of Cancer Trajectories: Background}

Cancer incidence and mortality rates, for most forms of cancer in most countries, increase at a declining rate with age, showing some leveling off before some age and, quite often, reaching a maximum and then declining $[10,16,21,26,38,50,58]$. The most common pattern shows cancer rates increasing at least up to age 75 , with marked leveling off or decline occurring, if 
at all, at more advanced ages where data may be unreliable. The second basic pattern shows a definite peak in cancer rates, either at a fairly young age and or in middle age, with declining rates after this peak.

The first pattern, with cancer rates increasing at a decreasing rate, can be modeled by a power (or Weibull) function of the form $\mu(x)=b x^{k}$, where $\mu(x)$ is the hazard rate of incidence or mortality at age $x$ and $b$ and $k$ are parameters [2,42]. In many cases, however, the incidence (or mortality) rates rise even less rapidly than a power curve and, as noted above, show a distinct leveling off and even decline at advanced ages. In an analysis of cancers with rates that generally increase with age, Cook [10] examined 338 data sets for 24 types of cancer in males, females, or both in eleven countries for ages 35 to 74: "In 54\% (181 out of 338), the lines showed downward curvature, that is, the rate of increase with age was less than predicted by [a power function]". Numerous other data sets, both for periods and for cohorts, also show a distinct leveling off of many cancer rates at older ages, especially at ages after 75 [21, 26, 50, 58].

The second basic pattern of cancer incidence and mortality rates shows a definite peak at an age at which data are generally considered to be reliable. Many nonepithelial tumors (such as leukemias or sarcomas) fit this pattern; the nonepithelial tumors, which account for only roughly 10 percent of fatal malignant tumors, may be produced by different mechanisms than epithelial tumors [44]. There are, however, also examples of carcinomas (i.e., epithelial tumors) that follow this second pattern. For instance, over the period 1973-77, the incidence of lung cancer peaked at about age 65 for U.S. females and the incidence of testicular cancer peaked at about age 25 for U.S. males. The incidence of cervical cancer among various cohorts of Danish, Finish, Icelandic, Norwegian, and Swedish women shows peaks at ages, depending on the cohort and the country, ranging from about 40 to about 60 [18]. In the case of the lung cancer, the pattern of sharp decline in period cancer rates (i.e., rates pertaining to some particular period of time, such as 1973-1977) can be largely explained by differences among cohorts, probably due to differences in smoking behavior [13]. Nonetheless, cohort patterns of lung cancer incidence and mortality also exhibit a definite leveling off and even some decline $[11,25,28,30,33,47,53]$.

The leveling and falling off of cancer rates is puzzling because the force of mortality for most causes of death and for all causes of death combined is most commonly modeled by an exponential (Gompertz) curve, at least until very advanced ages, and because theories of the causes of cancer suggest that the incidence of cancer should increase with age [6,22]. The observation that the age-trajectory of many cancer rates resembles a power function, at least before age 70 or so, led epidemiologists to develop various theories, most involving some multistage process, that imply a power function (or some similarly shaped trajectory) for cancer rates $[2,3,5,35,45,59]$. Which of these theories, if any, appropriately captures the etiology of cancer is, however, as yet unknown, given available ancillary theory and evidence, but it does seem likely that some kind of multistage process underlies the generation of most human cancers [44].

Numerous explanations have been suggested to explain the downward deviation of many cancer trajectories from the hypothesized power function pattern, including genetic heterogeneity among individuals in their susceptibility, environmentally acquired heterogeneity, age-related changes in insult, distortions in period data resulting from differences among cohorts, undercertification among the old, the effects of the time it takes a tumor to grow, and the effects of cellular event rates that are not small [37, 44]. Explanations based on the elimination of genetically-susceptible population groups have tended to be dismissed [10,34], in part because of the strong evidence, from migrant studies [20] and 
studies of the causes of lung cancer and other cancers [14], that behavioral and environmental factors must be very important.

Nonetheless, there appear to be substantial genetic differences among individuals, especially regarding genetic predisposition to environmental carcinogens [7, 23, 36, 43, 52], and the existence of strong genetic differences is not inconsistent with observed epidemiological patterns [45] or with the clear importance of environmental factors [23]. Moreover, even if susceptibility to some form of cancer is not influenced by genetic factors, susceptibility is influenced by various behavioral and environmental factors e.g., cigarette smoking, consumption of green vegetables, or exposure to asbestos that differ among the members of a population, leading to the formation, after birth, of more or less susceptible groups $[17,51]$. Indeed, multistage theories of cancer imply heterogeneity, because different individuals, even of the same age, will be at different stages.

Several alternative models that recognize heterogeneity among individuals in frailty and susceptibility yield population trajectories of cancer rates that rise, level off, and perhaps decline even though cancer trajectories for the individuals that comprise the population rise steadily [10, 27, 30, 32, 33, 45]; for more general reviews, see Economos [15], Keyfitz [24], and Vaupel and Yashin $[55,56]$.

Using cohort data on mortality from lung cancer among U.S. whites, Manton et al. [33] investigated twelve different models based on alternative assumptions about the age-specific pattern of mortality risks for individuals and about the distribution (if any) of mortality risks across individuals. For U.S. females, the best fitting model used an exponential function for the age trajectory of lung cancer mortality, although for U.S. males, the best fitting model used a power function. Employing the same approach to analyze total mortality from all causes for various U.S. cohorts, Manton et al. found the best fitting power function and exponential function models fit the data virtually equally well, for both female and male data. A lesson here is that it may be difficult to discriminate, using epidemiological data, between models based on different mortality functions, even though the different functions may have radically different theoretical implications about the etiology of cancer; Peto [45] discusses this. It should be noted that in Manton et al.'s analyses, models that assumed a considerable degree of population heterogeneity fit the data much better than models assuming homogeneity among individuals in mortality risks.

\subsection{The Rise and Fall of Cancer Trajectories: Some Simple Models}

One model of cancer mortality (or incidence) in a heterogeneous cohort is illustrated in Figure 1a; we will call this the prototypical susceptibility model. Some individuals are susceptible to some particular form of cancer (or, alternatively, to cancer in general); others are immune. This differential susceptibility could be the result of genetic, behavioral, or environmental factors. At starting age zero, which may be some age substantially later than birth to allow environmental risk factors to create the two sub-cohorts, a proportion $\pi_{0}$ are susceptible. For these individuals, the force of mortality from the cancer at age $x$ is $\mu_{c}(x)$; for both susceptible and immune individuals the force of mortality from other causes of death is $\mu_{0}(x)$. The observed force of mortality from the cancer for the population as a whole, $\bar{\mu}_{c}(x)$, is given by:

$$
\bar{\mu}_{c}(x)=\pi(x) \mu_{c}(x)
$$


where $\pi(x)$ denotes the proportion of the population alive at age $x$ that is susceptible to the cancer. This proportion is given by:

$$
\pi(x)=\frac{\pi(0) \exp \left(-\int_{0}^{x}\left(\mu_{c}(t)+\mu_{0}(t)\right) d t\right)}{\pi(0) \exp \left(-\int_{0}^{x}\left(\mu_{c}(t)+\mu_{0}(t)\right) d t\right)+(1-\pi(0)) \exp \left(-\int_{0}^{x} \mu_{0}(t) d t\right)}
$$

or, canceling and rearranging, by:

$$
\pi(x)=\left(1+\frac{1-\pi(0)}{\pi(0)} \exp \left(\int_{0}^{x} \mu_{c}(t) d t\right)\right)^{-1}
$$

The formula developed by Peto and presented in Cook et al. [10] as formula $(v)$ is, with appropriate change in notation and some rearrangement, equivalent to formula $\{3\}$. Note that the force of mortality from causes other than cancer does not appear in this formula and hence does not influence the cancer trajectory. Thus in this simple model, cancer and other causes of death are independent risks on both the individual and population levels.

Figure 2 a displays population trajectories of the cancer mortality rates, $\bar{\mu}_{c}$, produced by curves for the cancer mortality rates among susceptible individuals, $\mu_{c}$, given by a power function. The five curves in the figure, which differ because different parameter values were used in the underlying model, are reminiscent of observed trajectories of cancer mortality, as displayed, for instance, in Segi et al. [50] or Magnus [26]. Two parameters were used in drawing the curves $\pi_{0}=\pi(0)$, the proportion susceptible, and a parameter, called $C$, that gives the cumulative proportion of the susceptible population that would suffer this cancer by age 75 if they did not die of something else before then. This parameter $C$ can be interpreted as a measure of how much selection has taken place among the susceptible population by age 75 . For each of the curves, the power $k$ in the power function $\mu(x)$ was taken to be 6 , a frequently used value in cancer studies. Given $C$ and $k$, the scaling factor $b$ in the power function can be easily calculated. The proportion of the entire population that would suffer the cancer by age 75 is given by $\pi_{0}$ times $C$; for each of the five curves in the Figure $2 \mathrm{a}$ this product was held constant at 1 percent. Hence the curves can be interpreted as being determined by the five values chosen for $C$, namely 1 percent, 10 percent, 50 percent, 80 percent, and 100 percent. The curves thus illustrate how the impact of selection on a cancer trajectory depends on the degree of selection that has occurred. Experiments with other values of the product of $C$ and $\pi_{0}$ indicate that variation in how common the cancer is among the entire population has hardly any effect on the shape of the trajectories compared with the effect of changes in $C$ (at any constant level of $C$ times $\pi_{0}$ ).

Cook et al. [10] present, in their Figure 4, some curves similar to the ones in Figure 2a. They reject the importance of differential susceptibility, but their logic pertains to innate susceptibility rather than susceptibility acquired by behavior or environmental exposure and their evidence is based on some uncomplicated analysis of limited data pertaining to period rates for seven cancers in eleven countries, with a cutoff age of 74 . 
The prototypical susceptibility model in Figure 1a might be generalized to the prototypical frailty model shown in Figure 1b. Here the force of mortality from causes other than the cancer differs in the two subpopulations. If $\mu_{0}$ is greater than $\mu_{0}^{\prime}$, then the individuals in the first subpopulation might be called frail, because they are susceptible to the cancer and have a higher chance of death from other causes as well. This may be reasonable if risk factors associated with the cancer, whether genetic, behavioral or environmental, tend also to be associated with other causes of death or if persons with the cancer are weakened such that they have a higher chance of dying from other causes. For this frailty model, the death rate from the cancer in the population, $\bar{\mu}_{c}$, is given, as before, by $\{1\}$, but $\pi$ now depends on both $\mu_{c}$ and $\mu_{0}$ :

$$
\pi(x)=\left(1+\frac{1-\pi(0)}{\pi(0)} \exp \left(\int_{0}^{x}\left(\mu_{c}(t)+\mu_{0}(t)-\mu_{0}^{\prime}(t)\right) d t\right)\right)^{-1}
$$

The death rate from other causes in the population, $\bar{\mu}_{0}$, is given by

$$
\bar{\mu}_{0}(x)=\pi(x) \mu_{0}(x)+(1-\pi(x)) \mu_{0}^{\prime}(x)
$$

so now $\bar{\mu}_{c}$ and $\bar{\mu}_{0}$ are linked (via their common dependence on $\pi$ ): on the population level, the cancer and the other causes are not independent risks even though they are independent for every individual in the population. For instance, if $\mu_{c}$ decreases, then $\bar{\mu}_{c}$ will also decrease but $\bar{\mu}_{0}$ will increase.

This prototypical frailty model can produce, depending on the parameters used, population trajectories of cancer mortality that rise and fall or that rise and then level off before the cutoff age.

Some further directions for generalization of the susceptibility and frailty models are suggested in Figure 1c. Instead of being susceptible or immune, individuals might be more or less susceptible to the cancer under study. The parameters $\lambda$ and $\lambda^{\prime}$ represent intensities of flow from one state to the other. Individuals might become more susceptible to the cancer as a result of, e.g., cigarette smoking or exposure to radiation. On the other hand, individuals, by giving up cigarette smoking or changing jobs, might reduce their susceptibility. The force of mortality from other causes, $\mu_{0}$ and $\mu_{0}^{\prime}$, might also differ in the two states. If individuals who are susceptible to the cancer are frail or debilitated, $\mu_{0}$ might be greater than $\mu_{0}^{\prime}$. If, on the other hand, the risk factors that increase the chances of the cancer decrease the chances of, say, heart disease, then $\mu_{0}^{\prime}$ might be greater than $\mu_{0}$. In any case, in this model the two causes of death are not independent on the population level: a change in the observed force of mortality from the cancer will produce a change in the observed force of mortality from other causes and visa versa.

The model can be explored by running the following formulas and difference equations on a personal computer: 


$$
\begin{gathered}
p(0)=\pi(0), p^{\prime}(0)=1-\pi(0), \\
p(x+\Delta)=p(x)+\Delta\left[\lambda^{\prime}(x) p^{\prime}(x)-\left(\mu_{c}(x)+\mu_{0}(x)+\lambda(x)\right) p(x)\right], \\
p^{\prime}(x+\Delta)=p^{\prime}(x)+\Delta\left[\lambda(x) p(x)-\left(\mu_{c}^{\prime}(x)+\mu_{0}^{\prime}(x)+\lambda^{\prime}(x)\right) p^{\prime}(x)\right] \\
\pi(x)=p(x) /\left(p(x)+p^{\prime}(x)\right), \\
\bar{\mu}_{c}(x)=\pi(x) \mu_{c}(x)+(1-\pi(x)) \mu_{c}^{\prime}(x) .
\end{gathered}
$$

As an illustration of this general point, consider the model shown in Figure 1d. In this model, individuals are assumed, at starting age zero, to be immune from some cancer: people gradually become susceptible to the cancer at a constant rate $\lambda$. Susceptible individuals are assumed not only to be susceptible to the cancer being studied, but also to a related set of cancers. In terms of a multistage model, the transition from immune to susceptible can be considered a transition common to a group of cancers, a transition based on some environmental or behavioral factor that is a risk factor for all the various cancers in the group. This simple transition model was used to produce the curves shown in Figure $2 \mathrm{~b}$ : the more important other cancers are as a cause of death, the more bowed is the trajectory for the cancer being studied.

In addition to demonstrating that there are a variety of ways in which selection in a heterogeneous population can cause the age trajectory of cancer rates to level off or even decline, the various models presented above can be used to show that even substantial heterogeneity may produce only weak effects on age trajectories. Using the variant diagrammed in Figure 1e, Peto [45] demonstrates that even in cases where predisposed individuals - be they 1 percent, 10 percent or 50 percent of the total population - suffer 50 times the incidence of some cancer compared with the rest of the population, the age trajectory of cancer incidence in the population as a whole may closely mimic, at least up to age 75 or so, the age trajectory of a homogeneous population. Other variants of the model diagrammed in Figure 1c can produce similar results; some of the trajectories in Figures 2a and $2 \mathrm{~b}$ are examples. Thus, although heterogeneity may be a possible explanation for some of the observed leveling off and decline in cancer trajectories, the absence of apparent leveling off or decline does not imply homogeneity or even approximate homogeneity. This is reassuring because it is virtually certain that substantial heterogeneity exists, produced by various genetic, behavioral, and environmental risk factors, for cancers of all types. A key question that thus arises and which will be addressed in subsequent sections is the possible impact heterogeneity might have on time trajectories and geographic variations, even in those cases where the heterogeneity does not have a substantial effect on age trajectories.

More elaborate models, with many states of susceptibility or a continuous distribution of susceptibility and with susceptibility changing with age or time, can be developed [4, 31, 54, $57,60]$. In empirical analyses of particular forms of cancer or other causes of death, it may be appropriate to use models complicated enough to capture the key details of the disease process. Here our purpose is not statistical inference but insight. We will henceforth illustrate our examples with a single model that captures the key feature driving the process; it turns out that in all instances it is sufficient to use models as simple as the prototypical susceptibility model in Figure 1a, for which the risk of the cancer is independent of other mortality risks, or the prototypical frailty model in Figure $1 b$, for which the mortality risks are linked on the population level although they are independent for all individuals.

\subsection{Successive Apogees}


Figure 3a displays the age trajectories of incidence rates from cervical cancer for three birth cohorts of Icelandic women (redrawn from data in Hakama [18]). Similar patterns of crossover and successive apogees can be found for other cohorts, other countries, and other forms of cancer, e.g., for cervical cancer in Denmark [18], cancer of the larynx for French males [53], cancer of the bone among Japanese males and females, and leukemia among survivors of the atomic bomb attacks in Japan and lung cancer among insulation workmen [40].

The prototypical susceptibility model, shown in Figure 1a and described by formulas $\{1\}$ and $\{3\}$, yields successive apogees that resemble observed patterns. One simulation is presented in Figure $3 b$; in this simulation, the trajectories shift to the right as the mortality rate declines and the trajectories reach higher peaks as the proportion of the population susceptible to the cancer increases. As indicated above, a variety of other heterogeneity models, including models in which the observed rates of cervical cancer are increasing because competing causes of death are decreasing, can be used to produce trajectories similar to those in Figures 3a. We present Figure 3b, and the simple model underlying it, not as an explanation of cervical cancer trends in Iceland but as an illustration of the fact that such trends are consistent with the operation of selection in heterogeneous populations and hence might be partially accounted for by an appropriate heterogeneity model.

Although clear examples of successive mortality apogees for successive cohorts are unusual, perhaps because the age trajectories are usually cutoff at some age like 75 or 80 before the full effects of selection can be seen, examples of crossover or strong convergence are more common, as illustrated by the various cohort graphs in Segi et al. [50] and Magnus [26]. An interesting instance of a crossover in incidence rates is given in Hanai et al. [19] for stomach cancer among males and females in Osaka, Japan, who were classified into histologic groups. In addition, patterns of decline over time in age-specific rates at younger ages and increases at older ages are evident for all cancers combined and for various specific cancers in Japan [50] and other countries: Figure 4, which displays the age trajectories of death rates from cancer and from other causes among U.S. women in 1970 and 1980, shows a crossover for cancer at age 55 .

Differential selection in a heterogeneous population, which has been widely accepted as at least a partial explanation for mortality crossover and convergence in several areas [29, 56], may provide at least a partial explanation of these observations as well. In any case, under fairly general conditions [55], a decrease in mortality rates at younger ages will result, in a heterogeneous population, in an increase at older ages and a uniform decrease in the rates at all ages will result in an observed mortality trajectory that converges toward and may cross the previous trajectory. It follows, however, from Peto's [45] analysis, discussed above, that even strong heterogeneity and substantial cohort differences may not lead to noticeable convergence. Consequently, absence of convergence cannot be taken as evidence that the population is homogeneous.

\subsection{The Falling Cancer Share}

Death rates from cancer among U.S. women in 1980 rose more slowly with age than death rates from other causes. As a result, cancer accounted for a declining share of deaths with age: the share of deaths accounted for by cancer fell from 43 percent at ages 50-54 to only 9 percent at ages above 85 . In contrast, cardiovascular diseases accounted for 28 percent of deaths at ages 50-54 and fully 71 percent at ages above 85 . The declining cancer share and rising cardiovascular share, which can also be observed for males, other times, and other countries [46], is an implication of the fact that cancer rates tend to rise even more slowly than 
a power function, whereas cardiovascular disease death rates (and total death rates) rise exponentially. An interesting (and speculative) perspective on this can be developed by using a simple model, like the one in Figure 1a, that recognizes that people differ in their susceptibility to cancer. It may be that among people who are susceptible to cancer, the force of mortality from cancer is higher at all ages than the force of mortality from, say, cardiovascular disease. Indeed, the force of mortality from cancer among the susceptible population may be increasing exponentially. Nonetheless the observed age trajectory of cancer mortality may cross over the cardiovascular disease trajectory.

In the prototypical susceptibility model, the population force of mortality from cancer is given by formulas $\{1\}$ and $\{3\}$. Substituting $\{1\}$ into $\{3\}$ yields an integral equation for $\pi(x)$ :

$$
\pi(x)=\left(1+\frac{1-\pi(0)}{\pi(0)} \exp \left(\int_{0}^{x} \bar{\mu}_{c}(t) \pi(t) d t\right)\right)^{-1}
$$

Given the trajectory of $\bar{\mu}_{c}(x)$ and the value of $\pi(0)$, numerical methods can be applied to this equation to estimate the trajectory of $\pi(x)$. Then, by using $\{1\}$, the trajectory of $\mu_{c}(x)$ can be calculated. Thus, if the proportion of the population at age zero that is susceptible to cancer is specified, it is possible to estimate the force of mortality from cancer among the susceptible population. In the model the force of mortality from cardiovascular diseases is the same for all individuals, regardless of their susceptibility to cancer, so the individual and population death rates from cardiovascular disease will be identical.

Figure 5 was constructed by taking the observed cardiovascular disease and cancer mortality trajectories as given in the 1980 U.S. mortality statistics for females; it was assumed that 25 percent of the synthetic cohort experiencing these rates were susceptible to cancer at the starting age of 20. The observed age-specific death rate from cancer falls below the death rate from cardiovascular disease after age 60 . The model, however, indicates that the death rate from cancer among susceptible individuals exceeds the death rate from cardiovascular diseases at all ages on the graph. We emphasize that this result is presented as an illustration only; the model is simplistic and the data faulty: selection operates on real cohorts, not synthetic ones. The point, however, remains: the age trajectory of cancer mortality for susceptible individuals might rise considerable more quickly, reach considerably higher levels, and have a different shape than the observed population trajectory.

\section{Variation over Time and Place}

\subsection{Compensating Cancers}

The incidence of different kinds of cancer varies enormously, sometimes by several orders of magnitude, among various countries and regions [14, 38]. For all forms of cancer combined, the variation tends to be less. Table 1 presents the age-adjusted death rates for various forms of cancer and for cancer at all sites for the country that ranked 1st and the country that ranked 40th in a data set for 1978-9 compiled by the World Health Organization (and presented in [1]). (We chose rank 40 to avoid erroneous values associated with poor data collection and processing in some countries with very low reported rates; the data for the first ranked countries appeared fairly reliable, given its consistency with values reported for countries ranked 2 and 3). With the exception of leukemia, the ratio of the rates for the various cancers 
ranges from about 4 to about 9 , in contrast with a ratio of 2 for all cancers combined.

Apparently when some forms of cancer are unimportant, other forms grow in importance.

Table 1. Age-adjusted death rates per 100, 000 for various forms of cancer and for cancer at all sites for the 1 st and 40th ranked country.

\begin{tabular}{lcrrr} 
Cancer & Sex & $\begin{array}{r}\text { For 1 } \\
\text { ranked } \\
\text { country }\end{array}$ & $\begin{array}{r}\text { For 40 } \\
\text { ranked } \\
\text { country }\end{array}$ & Ratio \\
\hline Oral & M & 20.3 & 2.2 & 9.2 \\
Prostate & $\mathrm{F}$ & 7.2 & 0.8 & 9.0 \\
Lung & $\mathrm{M}$ & 41.0 & 5.4 & 7.6 \\
& $\mathrm{M}$ & 113.2 & 15.6 & 7.3 \\
Colon \& rectum & $\mathrm{F}$ & 30.9 & 5.0 & 6.2 \\
& $\mathrm{M}$ & 34.0 & 5.7 & 6.0 \\
Stomach & $\mathrm{F}$ & 29.5 & 7.7 & 3.9 \\
& $\mathrm{M}$ & 66.1 & 14.4 & 4.6 \\
Breast & $\mathrm{F}$ & 33.5 & 7.2 & 4.6 \\
Uterus & $\mathrm{F}$ & 33.8 & 7.9 & 4.3 \\
Leukemia & $\mathrm{F}$ & 30.0 & 7.5 & 4.0 \\
& $\mathrm{M}$ & 9.6 & 3.9 & 2.5 \\
All sites & $\mathrm{F}$ & 6.3 & 2.8 & 2.3 \\
& $\mathrm{M}$ & 275.0 & 135.0 & 2.0 \\
& $\mathrm{~F}$ & 172.3 & 86.9 & 2.0
\end{tabular}

Source: [1].

A similar phenomenon can be observed over time: in various countries as some forms of cancer have declined in importance, others have increased, so that total cancer rates tend to be more steady than rates from particular forms of cancer. In the United States, for instance, age-adjusted lung cancer mortality rates have increased substantially over recent decades, rates for stomach cancer and cancer of the uterus have substantially declined, and various other cancer rates have shown considerable fluctuation, but the overall cancer mortality rate has remained relatively steady, showing only a small rate of increase [1, 39].

Such compensating changes are consistent with a model that assumes people differ in their susceptibility to cancer (or some set of cancers). A simple version of such a model is shown in Figure 1f. The population death rates from the two forms of cancer, $\bar{\mu}_{c}^{l}(x)$ and $\bar{\mu}_{c}^{2}(x)$, are given by

$$
\bar{\mu}_{c}^{i}(x)=\pi(x) \mu_{c}^{i}(x), i=1,2
$$


where, following $\{3\}$, it can be shown that

$$
\pi(x)=\left(1+\frac{1-\pi(0)}{\pi(0)} \exp \left(\int_{0}^{x}\left(\mu_{c}^{1}(t)+\mu_{c}^{2}(t)\right) d t\right)\right)^{-1}
$$

Hence, the two trajectories for $\bar{\mu}_{c}^{i}(x)$ are linked. If $\bar{\mu}_{c}^{l}(x)$, say, declines, then $\pi(x)$ will grow and $\bar{\mu}_{c}{ }^{2}(x)$ will increase. Indeed, even if both $\mu_{c}{ }^{1}$ and $\mu_{c}{ }^{2}$ are declining, if more progress is being made against cancer 1 than cancer 2 , it may look, at ages old enough that selection has had a major impact, as if cancer 2 is increasing. Table 2 summarizes the results of a simulation using this model.

Table 2. Annual rates of progress in reducing death rates at various ages for cancer 1 , cancer 2 , and cancer 1 and 2 combined, for the susceptible subpopulation and the entire population

Annual rate of progress (in percent) in reducing death rate

\begin{tabular}{|c|c|c|c|c|c|c|} 
& \multicolumn{2}{|c|}{ from cancer 1 } & \multicolumn{2}{c|}{ from cancer 2 } & \multicolumn{2}{c|}{ from cancer 1 and 2 } \\
\hline & $\begin{array}{c}\text { Actual rate } \\
\text { for } \\
\text { susceptible } \\
\text { population } \\
\text { (in percent) }\end{array}$ & $\begin{array}{c}\text { Observed } \\
\text { rate for } \\
\text { entire } \\
\text { population } \\
\text { (in percent) }\end{array}$ & $\begin{array}{c}\text { Actual rate } \\
\text { for } \\
\text { susceptible } \\
\text { population } \\
\text { (in percent) }\end{array}$ & $\begin{array}{c}\text { Observed } \\
\text { rate for } \\
\text { entire } \\
\text { population } \\
\text { (in percent) }\end{array}$ & $\begin{array}{c}\text { Actual rate } \\
\text { for } \\
\text { susceptible } \\
\text { population } \\
\text { (in percent) }\end{array}$ & $\begin{array}{c}\text { Observed } \\
\text { rate for } \\
\text { entire } \\
\text { population } \\
\text { (in percent) }\end{array}$ \\
\hline 50 & 1 & 0.96 & 0.1 & 0.06 & 0.57 & 0.53 \\
60 & 1 & 0.88 & 0.1 & -0.02 & 0.57 & 0.44 \\
70 & 1 & 0.68 & 0.1 & -0.22 & 0.57 & 0.24 \\
80 & 1 & 0.25 & 0.1 & -0.65 & 0.57 & -0.18 \\
\hline
\end{tabular}

Source: Computer calculations using multiple susceptibility model, described in text with $\pi(0)=0.05$ and $\mu_{c}^{i}(x)=b_{1} x^{5}$, with $b_{i}$ chosen such that $\int_{0}^{75} \mu_{c}^{1}(x) d x=0.5$ and $\int_{0}^{75} \mu_{c}^{2}(x) d x=0.333$

At all ages, progress is being made in reducing mortality from cancer 2 at a rate of 0.1 percent per year. However, the observed rate of progress in reducing cancer 2 is much less than this and is negative at age 60 and subsequently. Note the magnitude of the observed negative rate of progress at ages 70 and 80 is 2.2 and 6.5 times the magnitude of the actual rate for susceptible individuals at these ages. The observed rate of progress in reducing mortality from cancer 1 is also less than the actual rate. This is another manifestation of the convergence and crossover of mortality trajectories discussed above: saving susceptible individuals at younger ages reduces observed rates of progress in reducing mortality at older ages. For both forms of cancer combined, the overall rate of progress among susceptible 
individuals is 0.57 percent per year at all ages, but the observed overall rate of progress is considerably less at ages 60 and 70 and at age 80 it appears as it the overall cancer death rate is increasing.

\subsection{Multiple Cancers}

The hypothesis that some individuals may be more susceptible than other individuals to several different forms of cancer (or to cancer in general) seems to run counter to the widely accepted view that, as Cairns [6] puts it, "each of the major cancers behaves almost as an independent entity; with few exceptions, people who have been successfully treated for one cancer are then neither more nor less likely in subsequent years to come down with a second, different variety." Schoenberg [48] reviews the evidence on multiple cancers and presents a detailed analysis of the Connecticut Tumor Registry. He concludes: "Results for non-simultaneously diagnosed malignant tumors from Connecticut indicate that individuals with one malignant neoplasm have 1.29 times the risk of developing a new independent primary tumor compared to individuals who never had cancer $(P<0.01)$. However, the increased risk of multiple primary tumors is highly selective on a site-specific basis". For example, women with breast cancer had 1.9 times the risk of cancer of the corpus uteri and 0.5 times the risk of cancer of the pancreas; men with lung cancer had 6.5 times the risk of cancer of the kidney and men with cancer of the colon had 0.5 times the risk of lung cancer.

To understand the relationship of statistics such as these to the heterogeneity models discussed in this paper, consider the model shown in Figure 1g. There are two kinds of cancer in the model and two kinds of individuals. One cohort of individuals is more susceptible to both kinds of cancer than the other cohort. For simplicity it is assumed that the two cancers have identical, independent incidence rates for each of the two groups. Death is ignored in the model because the concern is with multiple cancers among those who survive to some specified age.

Let $r$ denote the relative risk of incidence of either cancer for the more susceptible compared with the less susceptible group; we will call $r$ the relative risk of cancer incidence. Let $R$ denote the relative risk of cancer 2 among those who have developed cancer 1 compared with those who have not developed cancer 1 ; we will call $R$, which is essentially equivalent to the statistic used by Schoenberg, the relative risk of cancer 2 prevalence. The question is: what is the relationship of $R$ to $r$ ? The answer, surprisingly, is that the value of $R$ tells us very little about the value of $r$; and that $R$ can be fairly low (e.g., 1.29) when $r$ is extremely large or even infinite.

Let $n_{0}(x), n_{1}(x), n_{2}(x)$, and $n_{12}(x)$ denote the number of individuals in the less susceptible cohort at age $x$ who have no cancer, the first or the second cancer, or both, respectively, and use the same notation with a prime to denote the numbers for the more susceptible cohort. It is not difficult to show that for both groups:

$$
n_{i}(x)=\sqrt{n_{0}(0) n_{0}(x)}-n_{0}(x), i=1,2
$$

and

$$
n_{12}(x)=n_{0}(0)+n_{0}(x)-2 \sqrt{n_{0}(0) n_{0}(x)} .
$$


The value of $n_{0}(x)$ depends on the function chosen for the transition rate $\lambda$. If $\lambda$ is assumed to be a power function

$$
\lambda=b x^{k},
$$

then

$$
n_{0}(x)=n_{0}(0) \exp \left(-\frac{2 b}{k+1} x^{k+1}\right)
$$

and

$$
n_{0}^{\prime}(x)=n_{0}^{\prime}(0) \exp \left(-\frac{2 r b}{k+1} x^{k+1}\right)
$$

The value of $R(x)$, which is the ratio of the probability of cancer 2 given cancer 1 to the probability of cancer 2 given the absence of cancer 1 , can then be calculated by:

$$
R(x)=\frac{\frac{n_{12}(x)+n_{12}^{\prime}(x)}{n_{1}(x)+n_{1}^{\prime}(x)+n_{12}(x)+n_{12}^{\prime}(x)}}{\frac{n_{2}(x)+n_{2}^{\prime}(x)}{n_{0}(x)+n_{0}^{\prime}(x)+n_{2}(x)+n_{2}^{\prime}(x)}} .
$$

Table 3 presents the value of $R$ for various values of the relative risk of cancer incidence, $r$, suffered by persons in the more susceptible group. 
Table 3. The values of $R$, the relative risk of cancer 2 prevalence, at age 65 , for various values of $r$, the relative risk of cancer incidence, and, $\pi_{0}$ the proportion who are highly susceptible.

\begin{tabular}{rrr}
$\pi_{0}$ & $r$ & $R$ \\
\hline 0.5 & $\infty$ & 2.04 \\
0.5 & 100.0 & 2.00 \\
0.5 & 10.0 & 1.69 \\
0.5 & 3.0 & 1.25 \\
0.5 & 1.0 & 1.00 \\
0.1 & $\infty$ & 10.35 \\
0.9 & $\infty$ & 1.12 \\
0.5 & 3.3 & 1.29 \\
0.75 & 16.0 & 1.29 \\
0.78 & 100.0 & 1.29 \\
0.78 & $\infty$ & 1.29
\end{tabular}

Source: Calculations based on model described in text with $k=6$ and $b$ chosen such that $\int_{0}^{75} b x^{6} d x=0.1$ for the more susceptible group. Using a value of $k$ of 2 or 8 or a value of $b a$ hundred times lower or twice as high hardly alters the results.

As the table suggests, the value of $R$ is essentially independent of the value of $r$ when $r$ is 100 or more. When such a large differential exists between the more and less susceptible groups, $R$ is approximately equal to the inverse of the proportion of the population at age zero that is susceptible. Thus a value of $R$ of about 2 is consistent with a model in which about half the population is highly susceptible to both cancers. Even when $r$ is considerably less than 100 , the value of $r$ tends to be much larger than the value of $R$. For instance, a value of $R$ of 1.25 is consistent with a model in which half the population is three times more susceptible to both kinds of cancer than the other half of the population; if $R$ is 2 , then the more susceptible group could be a third of the total population with a relative risk of 10 or half of the total population with a relative risk of 100 or more.

A simple example helps provide an explanation of the difference between the relative risk of cancer incidence, $r$, and the relative risk of cancer 2 prevalence, $R$. Suppose half the population is highly susceptible to cancer 1 and 2 and the other half is essentially immune. More specifically, assume that the more susceptible group faces a risk of 2 percent of developing either cancer by age 65 whereas the risk for the less susceptible group is a thousand times less. If someone gets cancer 1 before age 65, that person is almost certainly a member of the highly-susceptible group and thus has a two percent chance of developing cancer 2 by age 65 . Because the chance of either cancer is low, the fact that someone does not have cancer 1 tells us very little about which group the person is in. Hence, since people 
without cancer 1 have about a fifty percent chance of being susceptible, their chance of having cancer 2 by age 65 is half of 2 percent. Thus, having cancer 1 only doubles the chance of cancer 2, even though having cancer 1 is very strong evidence that a person is highly susceptible and highly susceptible people face 1000 times the risk of cancer 1 or 2 .

For some pairs of cancers, people having one cancer seem to be less likely to have the second cancer than people without the first cancer [48]. This could imply that people highly susceptible to the first cancer have low susceptibility to the second cancer. Alternatively, however, it may be that people in one group are more susceptible to both kinds of cancer, given that they have neither cancer but that getting the first cancer lowers the chances of getting the second cancer, because the effects of treatment, of physiological or behavioral changes, or whatever.

As an example, consider again the model in Figure 1g, but now suppose that the four incidence rates, the $\lambda$ 's, on the bottom half of the diagram are less than the four $\lambda$ 's on the top half. Specifically, assume that the incidence of either of the cancers among people with the other cancer is some fraction of the incidence among people without the other cancer. Table 4 presents some values of the relative risk of cancer 2 prevalence, $R$, corresponding to various values of this fraction and various values of the relative risk of cancer incidence, $r$.

Table 4 . The value of $R$, the relative risk of cancer 2 prevalence at age 65 , for various values of $r$, the relative risk of cancer incidence $\lambda^{\prime} / \lambda$, the relative risk of incidence of second cancer, and $\pi_{0}$, the proportion who are highly susceptible.

\begin{tabular}{cccc}
$\pi_{0}$ & $\lambda^{\prime} / \lambda$ & $r$ & $\mathrm{R}$ \\
\hline 0.50 & 0.4 & $\infty$ & 0.82 \\
0.50 & 0.2 & $\infty$ & 0.41 \\
0.50 & 0.5 & 10 & 0.86 \\
0.25 & 0.5 & 3 & 0.78 \\
0.75 & 0.6 & $\infty$ & 0.81
\end{tabular}

Source: Computer calculations using numerical methods to approximate the model described in the text, with $k=6$ and $b$ chosen such that $\int_{0}^{75} b x^{6} d x=0.1$ for the more susceptible group.

The parameters used result in values of the relative risk of cancer 2 prevalence that are less than one. These values, which might be interpreted as indicating that people who are more susceptible to the first cancer are less susceptible to the second, were produced, however, by a model in which part of the population is more susceptible to both cancers.

The model of multiple cancer presented in Figure 1g is highly simplified. Furthermore, the evidence concerning multiple cancer must, as Schoenberg [48] discusses, be used with great caution. Nonetheless, the model and the evidence are perhaps sufficient to draw the 
conclusion that the current evidence about multiple cancer is not inconsistent with the existence of substantial differences among individuals in their susceptibility to groups of cancers and perhaps even to cancer in general.

\subsection{Why is Cancer Mortality Increasing over Time?}

As shown in Figure 6, the mortality rate from cancer among U.S. females aged 60-64 has remained fairly stable since 1900, whereas total mortality has fallen to close to a third of its 1900 level and cardiovascular mortality rates have been cut more than 50 percent since 1950 . Roughly similar patterns hold at other ages and for age-standardized rates, for males as well as females, although for males there is more of an increase in cancer rates, largely due to lung cancer. Much attention has been devoted to trying to determine trends in rates of cancer incidence and mortality, the causes of such trends, and the implications for policy [14, 26]. As explained in this section, heterogeneity makes such efforts even more difficult than previously recognized.

Before discussing why, it is useful to consider two other pieces of evidence. Figure 4 displays the age-specific U.S. female mortality trajectories for cancer and for all causes of death other than cancer for 1970 and for 1980. It is apparent that rapid progress has been made against causes of death other than cancer. For cancer, on the other hand, there is evidence of progress at younger ages and evidence of higher death rates at older ages.

If, instead of following a single country over time, a variety of countries are compared at some point in time, a pattern emerges that may be similar in origin to the increasing relative importance of cancer over time: the countries with the highest death rates from other causes tend to have the lowest death rates from cancer. For instance, using age-adjusted death rates for 1975 for 50 countries with widely varying rates ([50], Appendix Table I), the Spearman correlation coefficient between the rate for malignant neoplasms and the rate for all other causes of death is -0.27 for males and -0.39 for females. As in the case of temporal trends, it looks from this kind of cross-sectional data as if progress against other causes of death is associated with an increase in cancer mortality rates.

Hence, it may prove to be significant that observed cancer mortality rates for a population may decrease slowly, stay roughly level, or even increase even though the risks of cancer for the individuals that comprise the population are decreasing. There are three main mechanisms in heterogeneous populations that can produce such results.

(i) The Effect of Convergence and Crossover

A reduction in cancer mortality at all ages will, in a heterogeneous population, produce a mortality trajectory that converges toward and may cross the previous mortality trajectory. As a result, the observed death rate from cancer will decline, at all ages and especially at advanced ages, by less than the corresponding rate for individuals. At ages after the crossover, the reduction in the individual rates $\mu$ actually results in an increase in the observed rates $\bar{\mu}$.

Table 5 shows the trend in cancer mortality rates at various ages at the individual and population level based on the prototypical susceptibility model. Although among susceptible individuals cancer mortality rates are declining by 1 percent per year at all ages, the observed rate at age 70 is declining by less than half a percent per year and the observed rate at age 80 is increasing by more than half a percent per year. 
Table 5. Annual rate of progress in reducing cancer death rates at various ages, for the susceptible subpopulation and the entire population.

Annual rate of progress (in percent) in reducing cancer death rate:

\begin{tabular}{llr} 
Age & $\begin{array}{l}\text { Actual rate for } \\
\text { susceptible } \\
\text { subpopulation } \\
\text { (in percent) }\end{array}$ & $\begin{array}{r}\text { Observed rate for } \\
\text { entire population } \\
\text { (in percent) }\end{array}$ \\
\hline 50 & 1 & 0.96 \\
60 & 1 & 0.83 \\
70 & 1 & 0.44 \\
80 & 1 & -0.61
\end{tabular}

Source: Computer calculations using prototypical susceptibility model, described in text, with $\pi(0)=0.25$ and $\mu_{c}(x)=b x^{6}$, with $b$ chosen such that $\int_{0}^{75} \mu_{c}(x)=0.8$.

\section{(ii) The Effect of Compensating Cancers}

Progress against one form of cancer may be offset by an observed rise in other forms for which susceptible individuals face higher risks. As illustrated above in Table 2, even if progress is being made against all forms of cancer, if the rates of progress differ for different forms of cancer, it may appear as if certain cancers are growing in importance. The observed death rate, at any age and especially at older ages, from all forms of cancer will, as a result, decline more slowly than the actual death rate among susceptible individuals.

\section{(iii) The Effect of Progress Against Other Causes}

A similar effect will result if individuals who are susceptible to cancer are also more susceptible to other forms of death, as in the prototypical frailty model. In this model, the observed age-specific death rates from cancer and other causes are linked as described by formulas $\{4\}$ and $\{5\}$. The formulas imply that a proportional decrease in the death rates from other causes among the frailer and more robust subcohorts may result in an increase in the observed death rates from cancer, even though the death rates from cancer among susceptible individuals remain the same. Indeed, even if the death rates from cancer among susceptible individuals decrease somewhat, the observed death rates from cancer may increase.

The statistics in Table 6 provide an illustration. 
Table 6. Annual rates of progress in reducing death rates at various ages for cancer and other causes of death, for the frail and robust populations and for the entire population.

Annual rate of progress (in percent) in reducing death from:

\begin{tabular}{lrr|rr}
\multicolumn{2}{c|}{ Cancer } & \multicolumn{2}{c}{ Other causes } \\
\hline Age & $\begin{array}{r}\text { Actual rate for } \\
\text { frail and robust } \\
\text { subpopulations } \\
\text { (in percent) }\end{array}$ & $\begin{array}{r}\text { Observed rate } \\
\text { for entire } \\
\text { population } \\
\text { (in percent) }\end{array}$ & $\begin{array}{r}\text { Actual rate for } \\
\text { frail and robust } \\
\text { subpopulations } \\
\text { (in percent) }\end{array}$ & $\begin{array}{r}\text { Observed rate } \\
\text { for entire } \\
\text { population } \\
\text { (in percent) }\end{array}$ \\
\hline 50 & 0.25 & 0.18 & 1.0 & 0.99 \\
60 & 0.25 & 0.04 & 1.0 & 0.97 \\
70 & 0.25 & -0.36 & 1.0 & 0.96 \\
80 & 0.25 & -0.38 & 1.0 & 0.98
\end{tabular}

Source: Computer calculations using prototypical frailty model, described in the text, with $\pi(0)=0.25, \mu_{0}(x)=0.0001 e^{0.1 x}, \mu_{c}(x)=b x^{5}$ and $b$ chosen such that $\int_{0}^{75} \mu_{c}(x) d x=0.8$, and $\mu_{0}^{\prime}(x)=0.5 \mu_{0}(x)$.

On the individual level, mortality rates from cancer are being reduced, at all ages, by a quarter of a percent per year and mortality rates from other causes are being reduced by one percent per year. The observed rate of progress against cancer, however, is close to zero at age 60; at ages 70 and 80 it looks as if there is a substantial rate of increase in cancer mortality. Because frail individuals are also at higher risk from other causes of death, the operation of differential selection also results in an observed rate of progress against other causes of death that is lower than the actual rate on the individual level, but the effect here is minor.

\section{Discussion}

Individuals differ in their chances of different causes of death: epidemiologists spend much of their time trying to uncover the risk factors that underlie these differences. Yet nearly all studies of competing causes of death assume that all individuals of the same age in the same population group face the same risks and that these risks are independent of each other. The first assumption is clearly invalid and, as we have illustrated in this article, the second assumption is invalid for heterogeneous populations, except in some special circumstances. Even if different causes of death are independent for individuals in some state (e.g., for frail individuals), they will not, in general, be independent for the population as a whole.

We have illustrated the potential impact of heterogeneity and dependent competing risks by using the various puzzles and paradoxes of cancer mortality to motivate the analysis. Our goal has been insight and conceptual advance, not empirical analysis or statistical inference. That work, clearly important, has been started elsewhere (especially by Manton and his colleagues). It will undoubtedly grow in significance as more and more realistic models are developed for particular disease contexts and as the paradigm shift continues toward recognition of the significance of differential selection in epidemiology, demography, 
actuarial mathematics, reliability engineering, and other population disciplines. Examples of the application of heterogeneity modeling to phenomena as diverse as fertility, divorce, the extinction of families of species, equipment failure, dental caries, accident proneness and the discovery of new petroleum deposits are reviewed in Vaupel and Yashin [55, 56].

This paper began with a list of seven puzzles and paradoxes of cancer epidemiology. Its findings can be summarized using the same list. The effects of differential selection in a population in which individuals vary in their susceptibility to cancer in general, or to various forms of cancer, or to various causes of death may partially account for:

- the leveling off and decline of cancer incidence and mortality rates with age,

- the fact that a greater proportion of deaths are from cancer around ages 50 or 60 than at older ages,

- the greater variation among countries in incidence and mortality rates from various forms of cancer than in incidence and mortality rates from cancer in general,

- the convergence and crossover of the age trajectories of cancer rates for two cohorts, from different time periods, countries, histological types, etc.,

- the negative correlation among countries in the mortality rates from cancer vs. other causes of death,

- the increase over time in mortality for some forms of cancer and decrease for other forms, and

- the tendency for cancer incidence and mortality rates to somewhat increase, whereas mortality rates from almost all other major causes of death, including heart disease and cerebrovascular disease, are declining.

In addition, two other findings are worth highlighting.

First, a variety of different heterogeneity models can produce qualitatively similar mortality patterns and trajectories. Simple susceptibility and frailty models that only distinguish two kinds of individuals were sufficient to illustrate the points made in this paper. It seems likely, though, that more elaborate models will fit empirical data better than such simple models. Even if some heterogeneity models, e.g. the prototypical susceptibility model, can be ruled out because of evidence, say, that few if any individuals are completely immune to cancer, other, more elaborate models may still be plausible. Indeed, individuals differ from each other along so many important risk factors that it seems incontrovertible that populations are heterogeneous: the question is when does this heterogeneity have an important effect on epidemiological statistics and, when it does, how should the heterogeneity be modeled?

Second, even substantial differences among individuals in their susceptibility to cancer or to some form of cancer may not produce a noticeable impact on the observed age trajectory of cancer mortality in a population. The impact of such differences, however, may significantly alter patterns of cancer mortality over time or place. Therefore, the absence of strong heterogeneity effects in some set of cancer statistics should not be assumed to exclude strong effects in other sets of cancer statistics.

These various findings open up new hypotheses and new research directions. Even less is known about cancer epidemiology than was previously thought, but more can be learned by systematically considering the effects of differential selection in heterogeneous populations. Some key facts that were assumed self-evident - such as the belief that little or no progress is 
being made in reducing rates of overall cancer mortality - may still be correct, but that remains to be established. 


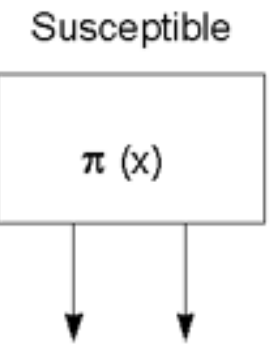

Immune

$\mu_{c}(x) \quad \mu_{o}(x)$

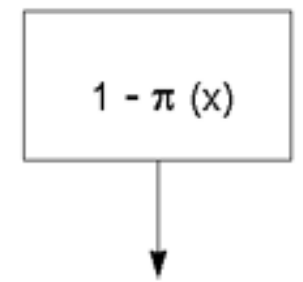

Fig 1a: The prototypical susceptibility model

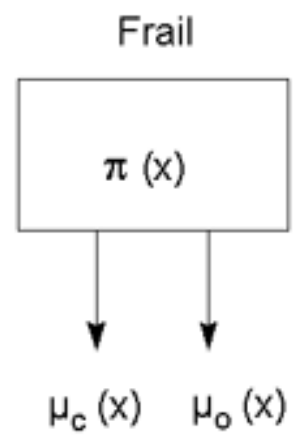

Robust

Fig 1b: The prototypical frailty model

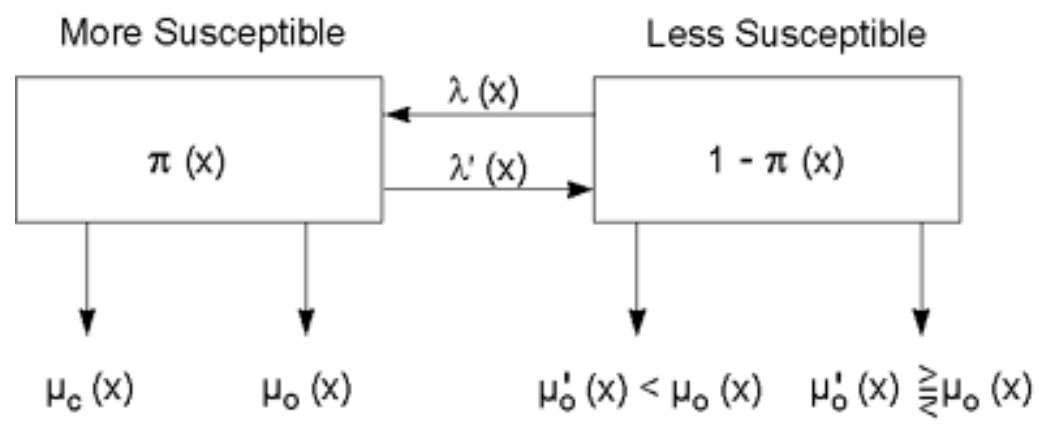

Fig 1c: A more general model

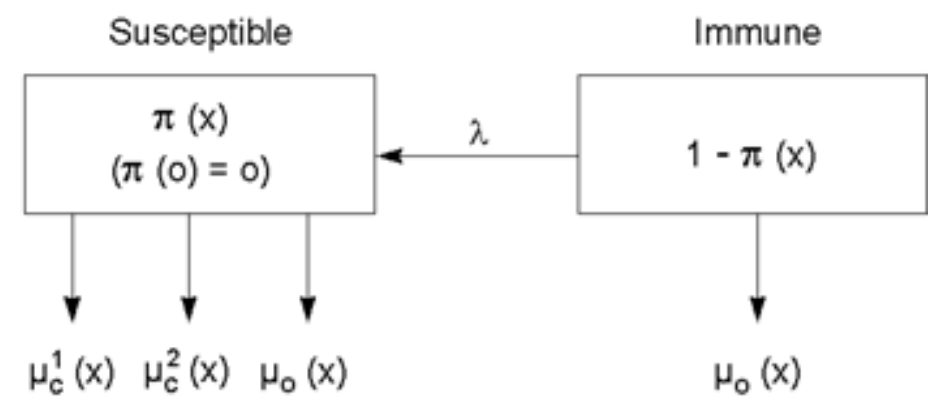

Fig 1d: The two-cancer transition model 

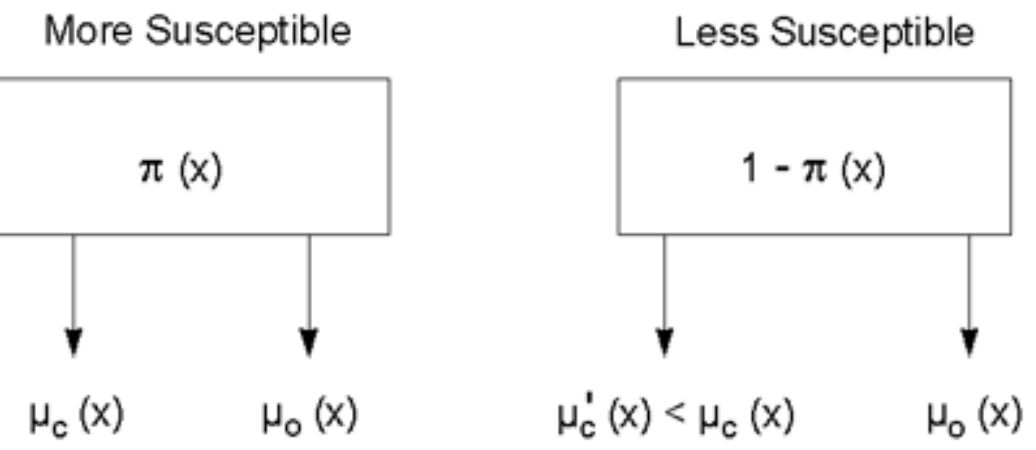

Fig 1e: The differing susceptibility model

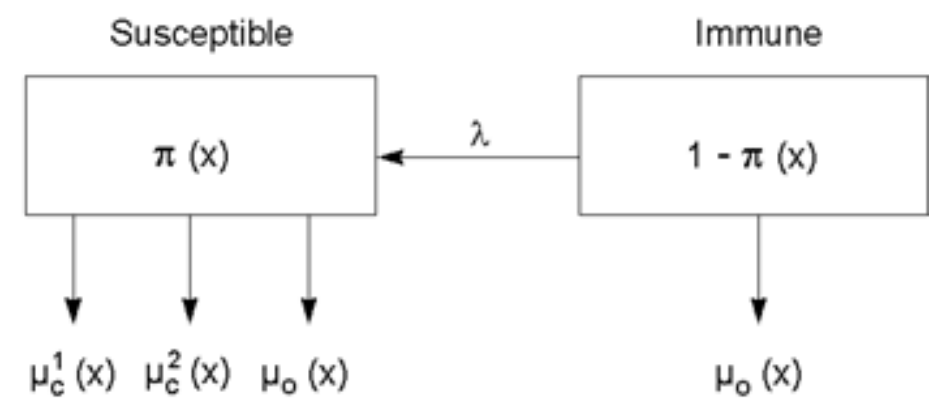

Fig 1f: The multiple cancer model
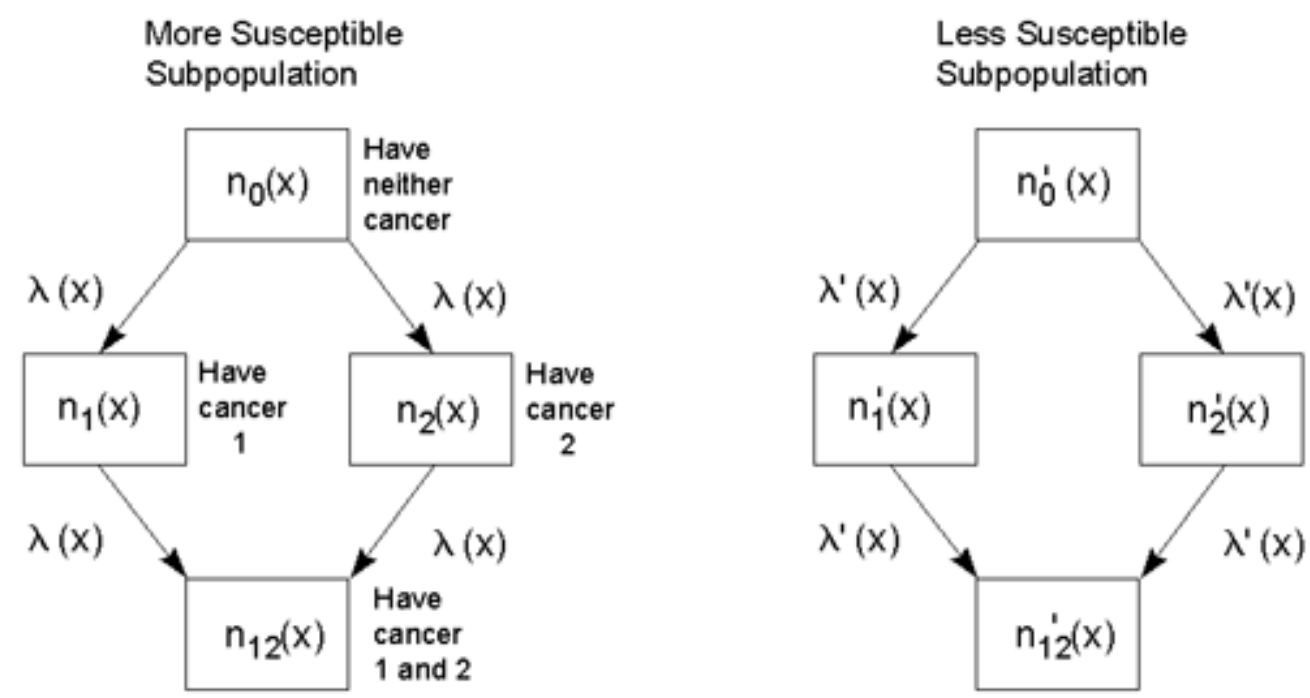

Fig 1g: The multiple cancer model 


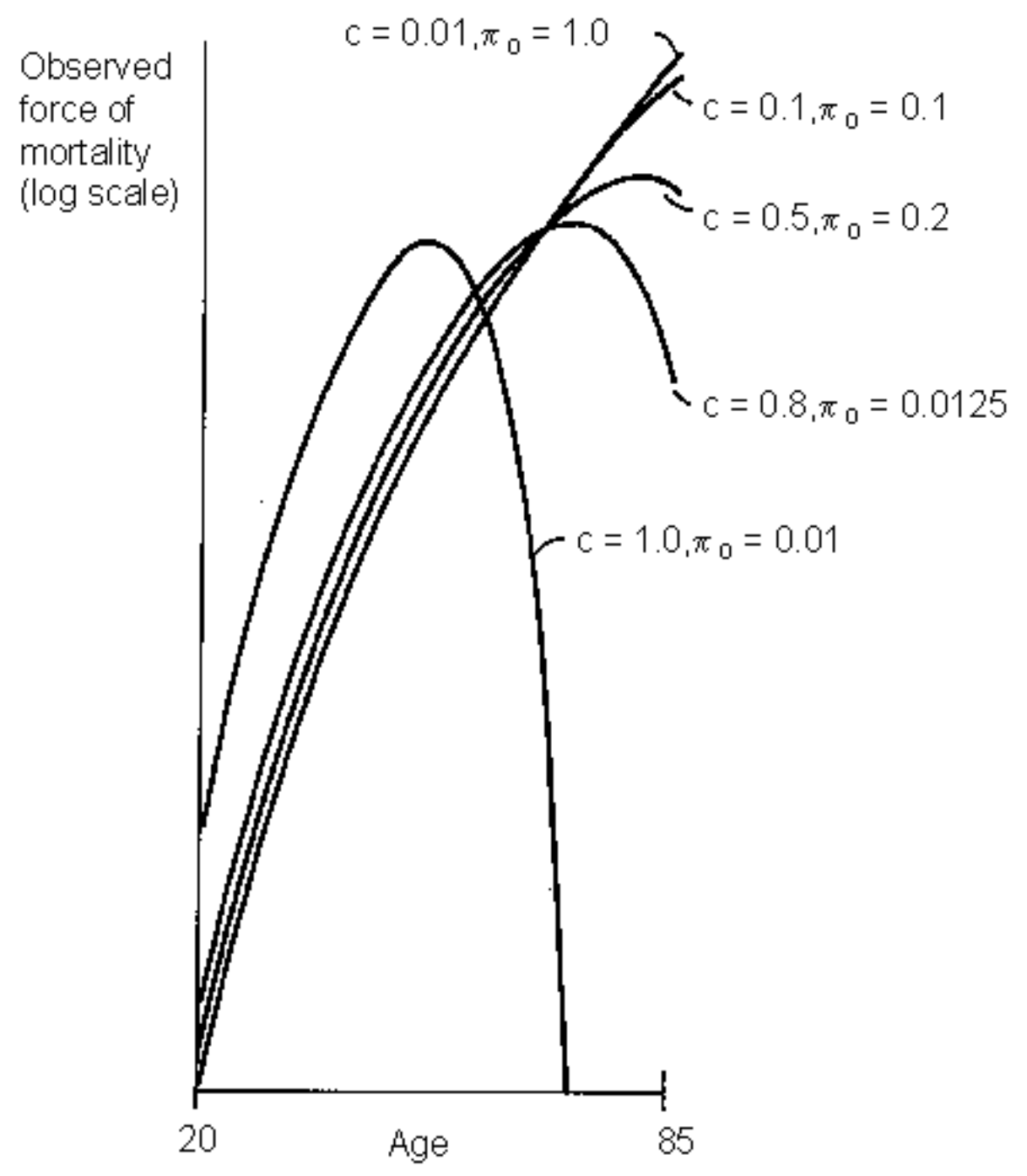




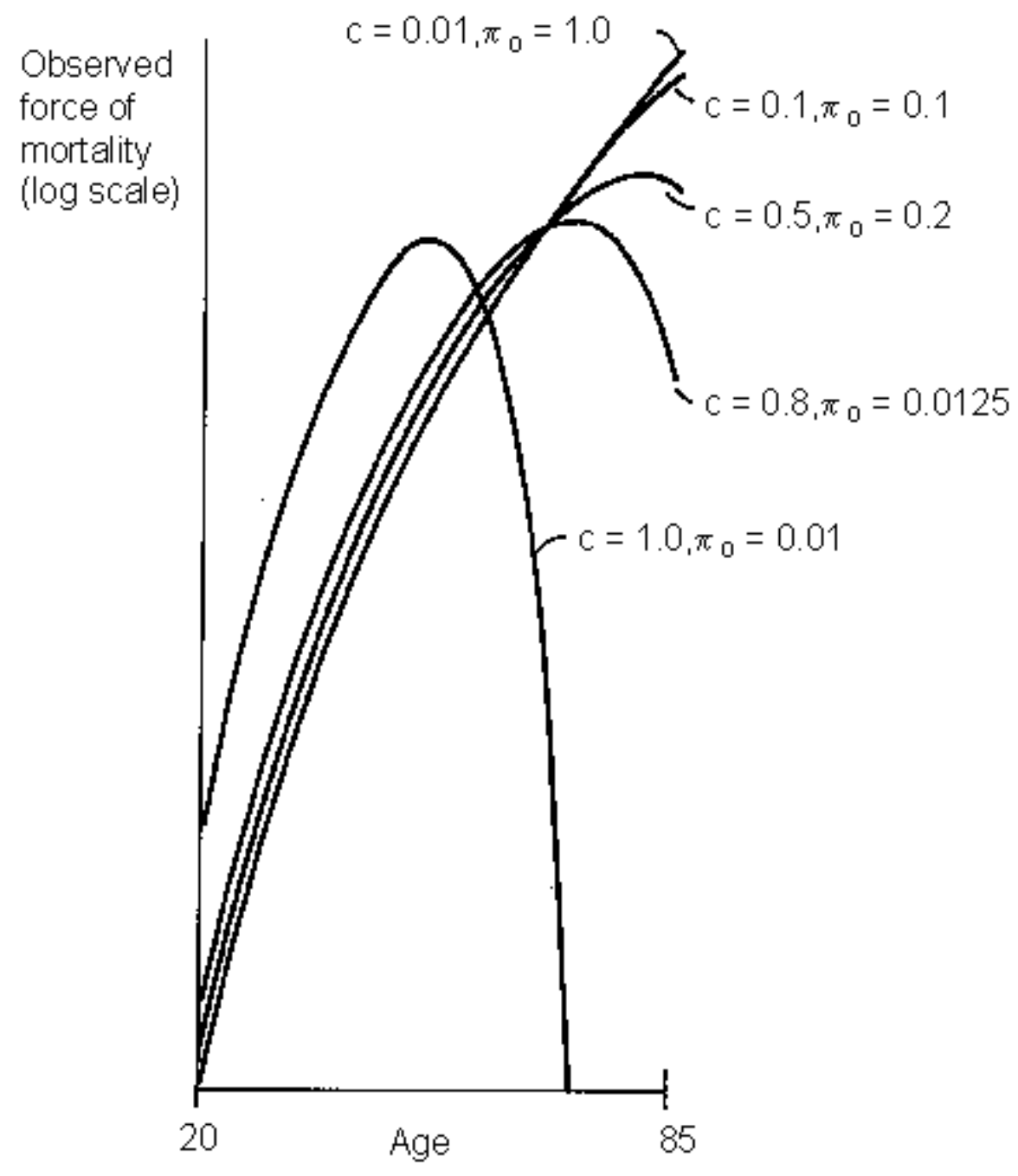

Figure 2a.

The observed force of mortality from cancer from age 20 to 85 produced by the prototypical susceptibility model for 5 pairs of parameter values. See text for details. 


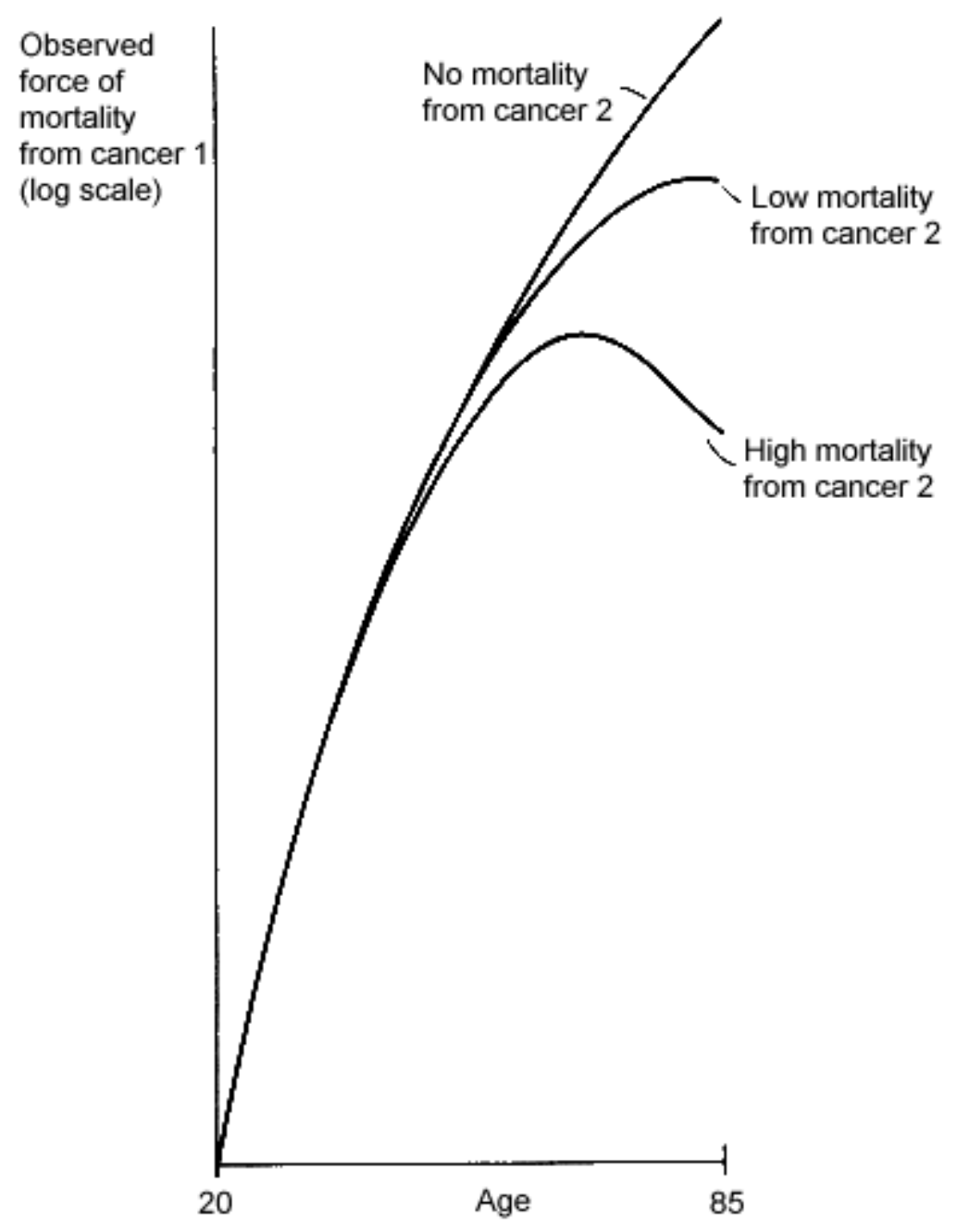

Figure 2b.

The observed force of mortality from cancer 1 from age 20 to 85 produced by twocancer transition model for three levels of mortality from cancer 2 . See text for details of the model. The following parameters were used: $\lambda=0.01, \mu_{c 1}(x)=b x^{5}$ with $\mathrm{b}$ chosen such that $\int_{0}^{75} \mu_{c 1}(x) d x=0.01 ; \mu_{c 2}(x)=b x^{5}$ with $\mathrm{b}$ chosen such that $\int_{0}^{x} \mu_{c x}(x) d x=0,0.5$ and 0.9 , respectively, for the three curves, and $\mu_{0}(x)=0.0001 e^{.1 x}$. 


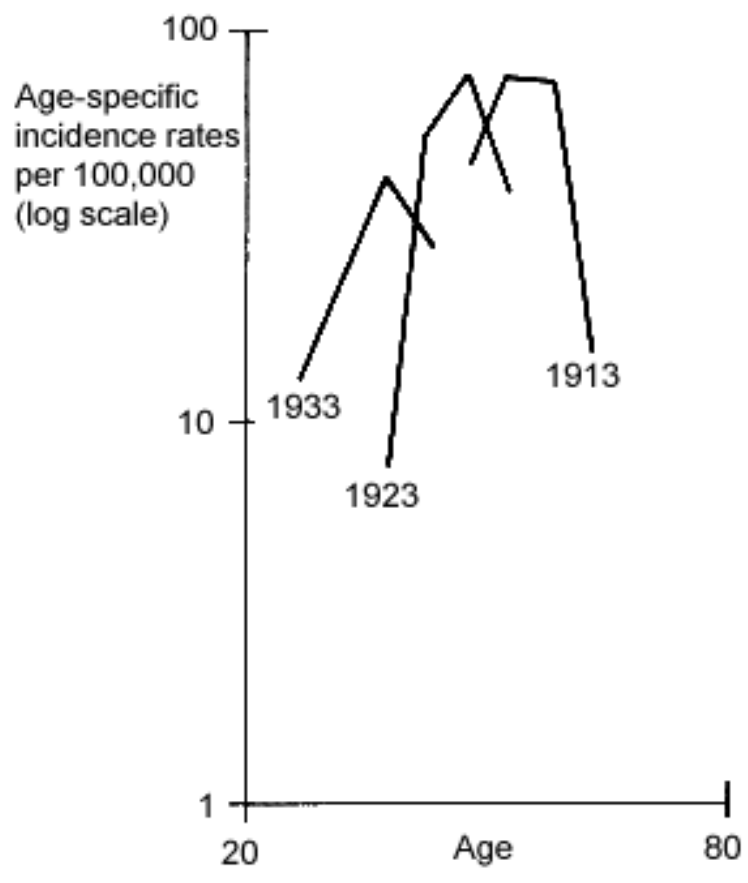

Figure 3a.

Age-specific incidence rates from cervical cancer for cohorts of Icelandic women born in 1913, 1923, and 1933. Adopted from [18]. 


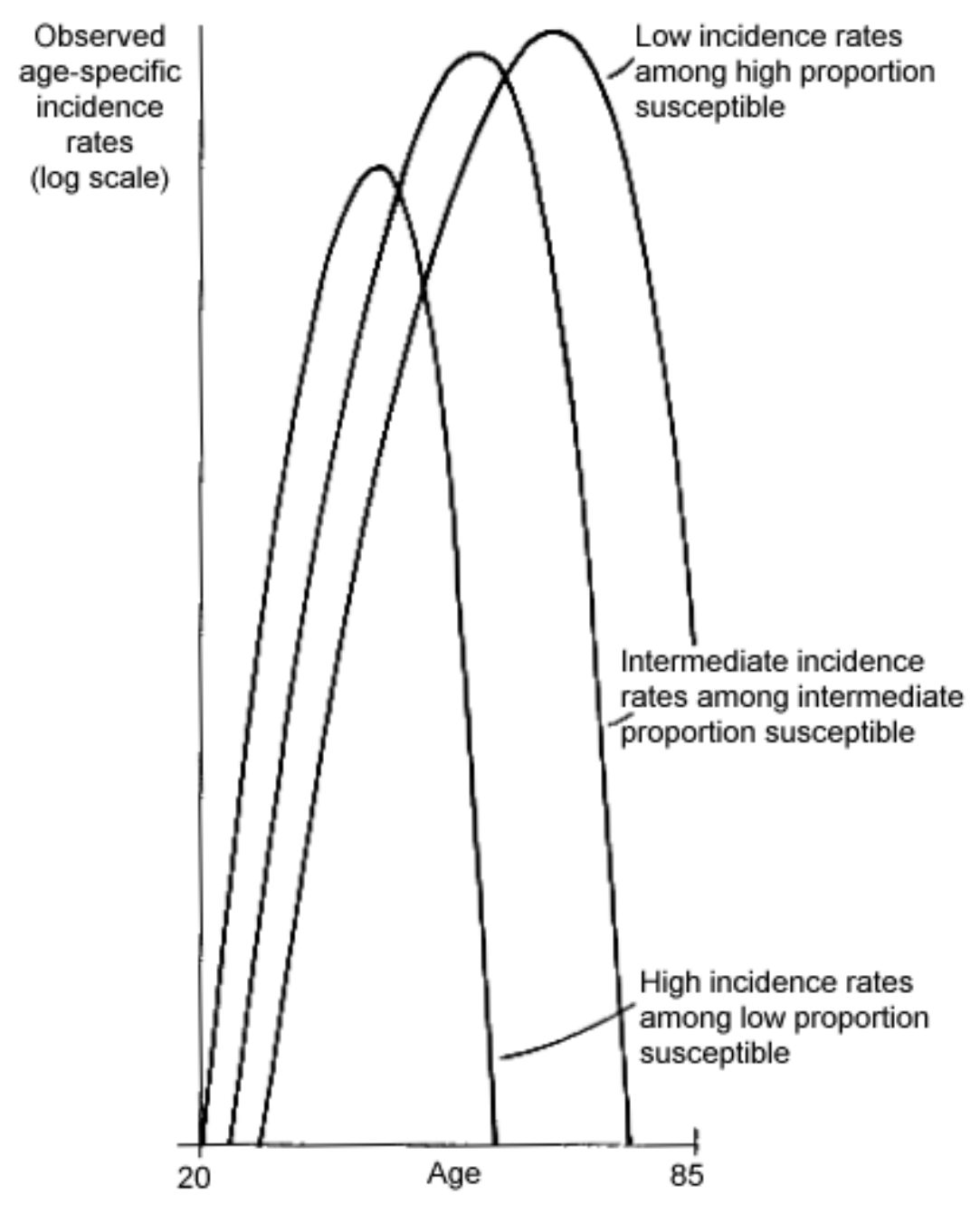

Figure 3b.

Age-specific incidence rates from age 20 to 85 produced by the prototypical susceptibility model for three sets of parameter values. See text for details of model. For the three curves from left to right $\pi_{0}$ is $0.012,0.02$, and 0.022 and $\mu(x)=a x^{6}$, where $\mathrm{a}$ is chosen such that $\int_{0}^{t} \mu(\mathrm{x}) \mathrm{dx}=1,0.99$, and 0.9 for $t=65,70$, and 75 , respectively. 


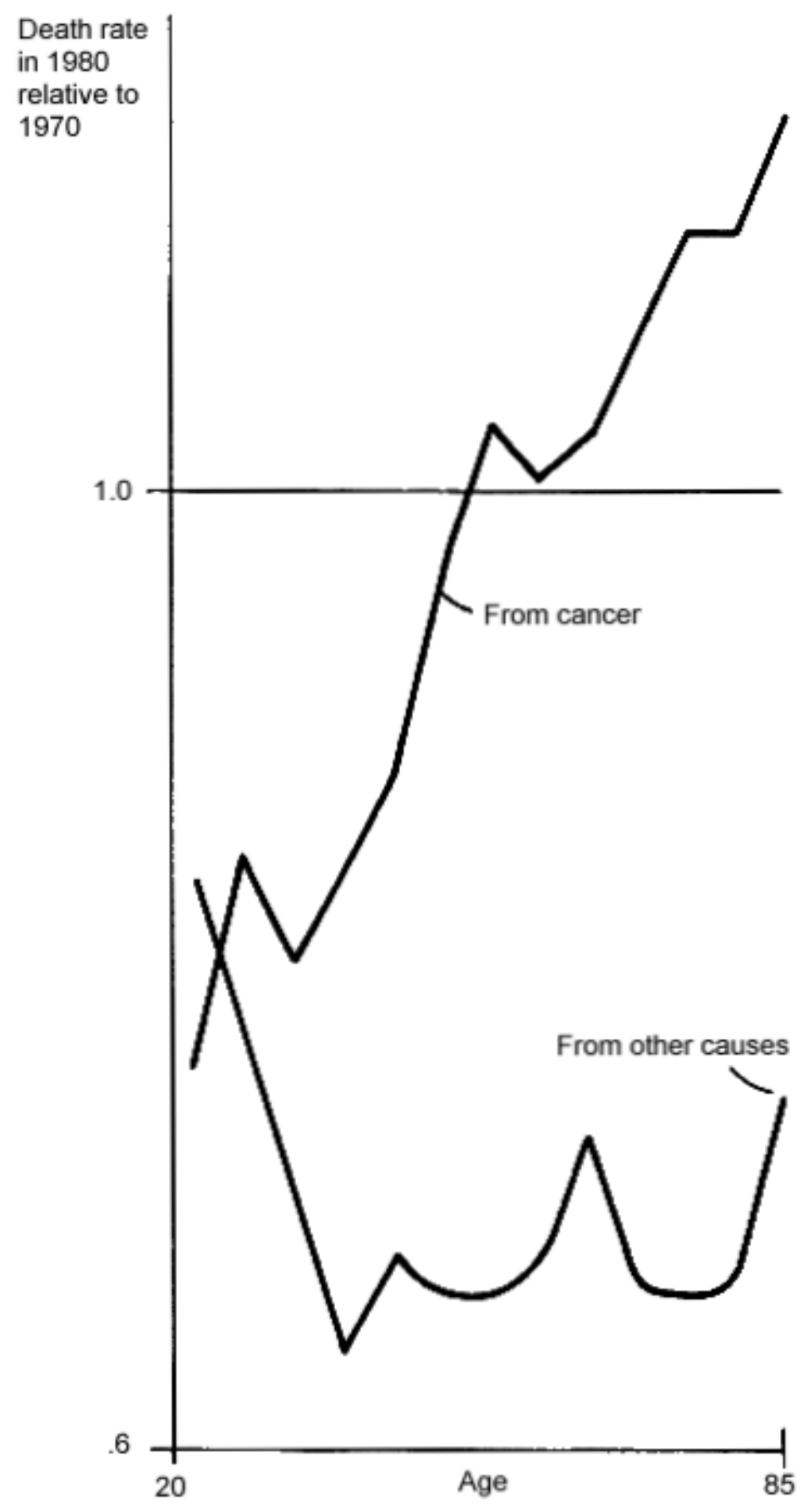

Figure 4.

Death rates in 1980 relative to 1970 from cancer and other causes for U.S. women from age 20 to 85 . 


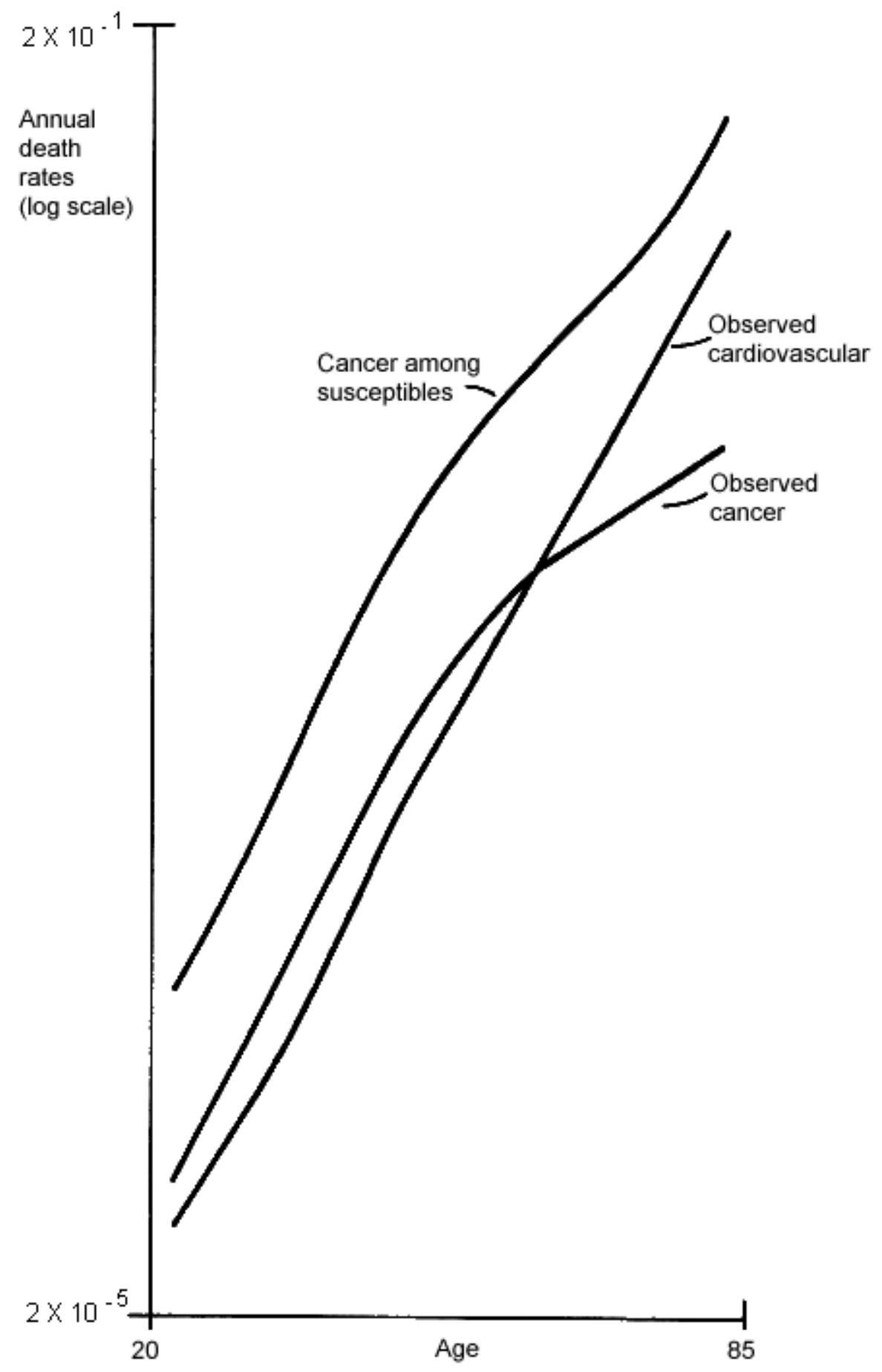

Figure 5.

Annual death rates from cancer and cardiovascular diseases in 1980 for U.S. women from age 20 to 85 . The death rates from cancer among susceptibles were calculated as described in the text, with $\pi_{0}$, the proportion susceptible at the starting age (of 20) equal to 0.25 . 


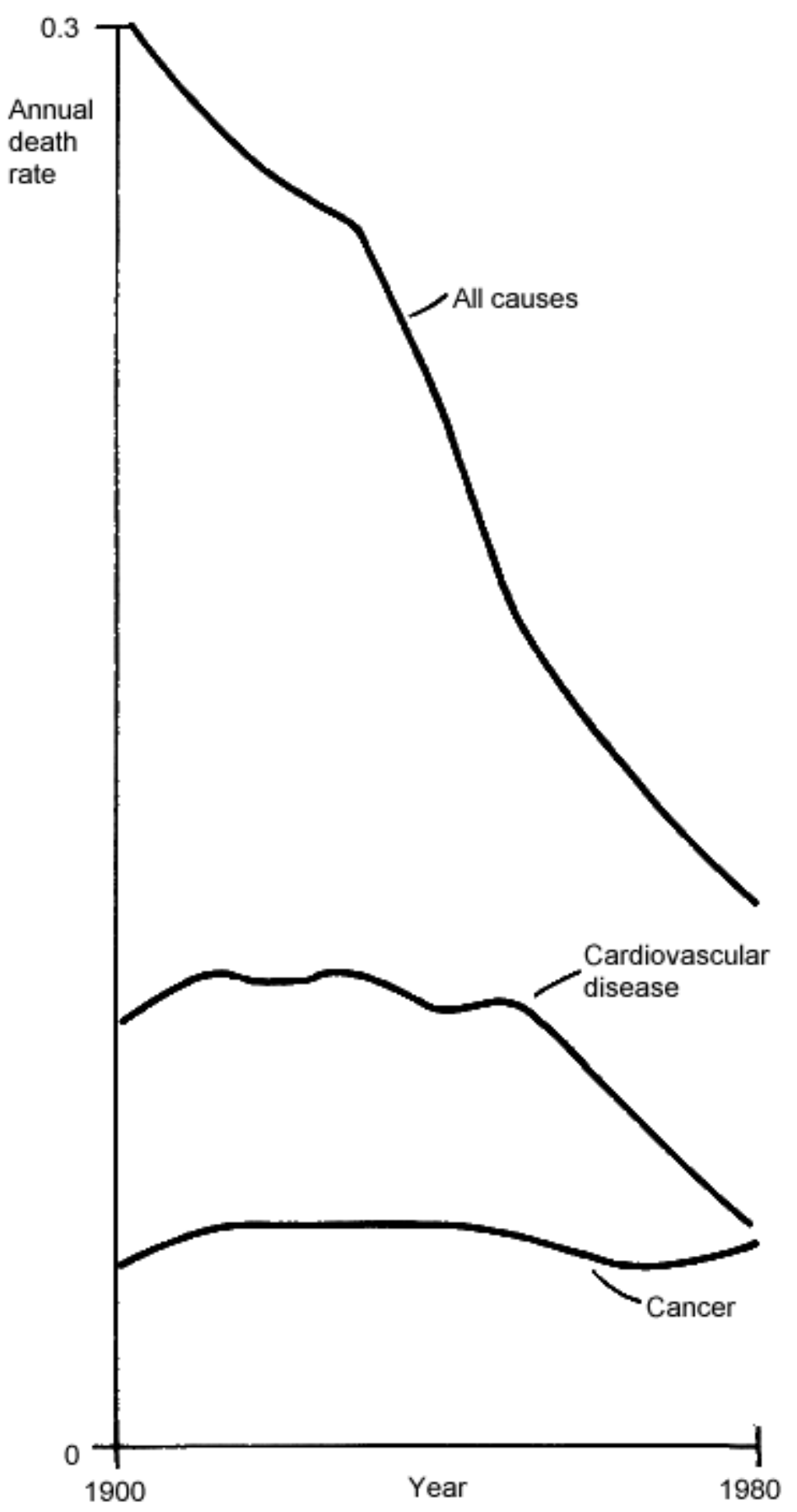

Figure 6.

Annual death rates from all causes, cardiovascular disease, and cancer from 1900 to 1980 for U.S. women aged 60 - 65. 


\section{References}

1. American Cancer Society. Cancer Facts and Figures. New York: American Cancer Society; 1985.

2. Armitage P, Doll R. Age Distribution of Cancer. British Journal of Cancer 1954;8:1-12.

3. Armitage, P, Doll R. Stochastic models for carcinogenesis. Proceedings of the Fourth Berkeley Symposium on Mathematical Statistics and Probability. Biology and problems of health, vol IV. Berkeley: University of California Press; 1961. p. 19.

4. Beard RJ. A theory of mortality based on actuarial, biological, and medical considerations. Proceedings of the International Population Conference. London: International Union for the Scientific Study of Population; 1963. p. 257.

5. Burch PRJ. Natural and radiation carcinogenesis in man: 1. theory of initiation phase. Proceedings of the Royal Society B 1965;162:223-39.

6. Cairns J. Cancer: Science and society. San Francisco: Freeman; 1978.

7. Cairns J, Lyon JL, Skolnick M, editors. Cancer incidence in defined populations. Banbury Report; 4. Cold Spring Harbor, NY: Cold Spring Harbor Laboratory; 1980.

8. Chiang CL. Introduction to stochastic processes in biostatistics. New York: Wiley; 1968.

9. Cohen J, Liu L. Competing Risks Without Independence. Manuscript. 1984.

10. Cook PJ, Doll R, Fellingham SA. A mathematical model for the age distribution of cancer in man. International Journal of Cancer 1969;4(1):93-112.

11. Day NE, Charnay B. Time trends, cohort effects, and aging as influence on cancer incidence. In: Magnus K, editor. Trends in cancer incidence: causes and practical implications. Proceedings of a symposium; 1980 Aug 6-7; Oslo, Norway. Washington: Hemisphere Publishing; 1982. p. 51.

12. DeVita VT Jr, Hellman S, Rosenberg SA, editors. Cancer: principles and practice of oncology. $2^{\text {nd }}$ ed. Philadelphia: Lippincott; 1985.

13. Doll R. The epidemiology of cancer. Cancer 1980;45(10):2475-85.

14. Doll R, Peto R. The causes of cancer: quantitative estimates of avoidable risks of cancer in the United States today. Journal of the National Cancer Institute 1981;66(6):1191-308.

15. Economos AC. Rate of aging, rate of dying and the mechanism of mortality. Archives of Gerontology \& Geriatrics 1982;1(1):3-27.

16. Engel A, Larsson T, editors. Cancer and aging. Proceedings of the Thule International Symposium on Cancer and Aging; 1967 Sep 26-28; Stockholm, Sweden. Stockholm: Nodriska Bokhandeln; 1968.

17. Fraumeni JF Jr, editor. Persons at high risk of cancer: an approach to cancer etiology and control. Proceedings of a conference; 1974 Dec 10-12; Key Biscayne, Florida. New York: Academic Press; 1975.

18. Hakama M. Trends in the incidence of cervical cancer in the Nordic countries. In: Magnus $\mathrm{K}$, editor. Trends in cancer incidence: causes and practical implications. Proceedings of a symposium; 1980 Aug 6-7; Oslo, Norway. Washington: Hemisphere Publishing; 1982. p. 279. 
19. Hanai A, Fujimoto I, Taniguchi H. Trends of stomach cancer incidence and histological types in Osaka. In: Magnus K, editor. Trends in cancer incidence: causes and practical implications. Proceedings of a symposium; 1980 Aug 6-7; Oslo, Norway. Washington: Hemisphere Publishing; 1982. p. 143.

20. Hanszel W. Migrant studies. In: Schottenfeld D, Fraumeni JF Jr, editors. Cancer epidemiology and prevention. Philadelphia: Saunders; 1982. p. 194-207.

21. Heston JF et al [Cusano MM, Young JL Jr, editors]. Forty-five years of cancer incidence in Connecticut: 1935-79. National Cancer Institute Monograph; 70. Washington, DC: for sale only by the Supt. of Docs., U.S. G.P.O.; 1986. p. 1.

22. Hiatt HH, Watson JD, Winsten JA, editors. Origins of human cancer. Book C: Human risk assessment. Cold Spring Harbor conferences on cell proliferation; 4. Proceedings of a conference; 1976 Sep. Cold Spring Harbor, NY: Cold Spring Harbor Laboratory; 1977.

23. Knudson AG Jr. Genetic predisposition to cancer. In: Hiatt HH, Watson JD, Winsten JA, editors. Origins of human cancer. Book C: Human risk assessment. Cold Spring Harbor conferences on cell proliferation; 4. Proceedings of a conference; 1976 Sep. Cold Spring Harbor, NY: Cold Spring Harbor Laboratory; 1977. p. 45.

24. Keyfitz N. Applied mathematical demography. $2^{\text {nd }}$ ed. New York: Springer; 1985.

25. Kvaale G. Incidence trends in the nordic countries. In: Magnus K, editor. Trends in cancer incidence: causes and practical implications. Proceedings of a symposium; 1980 Aug 6-7; Oslo, Norway. Washington: Hemisphere Publishing; 1982. p. 185.

26. Magnus K, editor. Trends in cancer incidence: causes and practical implications. Proceedings of a symposium; 1980 Aug 6-7; Oslo, Norway. Washington: Hemisphere Publishing; 1982.

27. Manton KG, Stallard E. A two-disease model of female breast cancer: mortality in 1969 among white females in the United States. Journal of the National Cancer Institute 1980;64(1):9-16.

28. Manton KG, Stallard E. A population-based model of respiratory cancer incidence, progression, diagnosis, treatment, and mortality. Computers \& Biomedical Research 1982;15(4):342-60.

29. Manton KG, Stallard E. Methods for evaluating the heterogeneity of aging processes in human populations using vital statistics data: explaining the black/white mortality crossover by a model of mortality selection. Human Biology 1981;53(1):47-67.

30. Manton KG, Stallard E. Bioactuarial models of national mortality time series data. Health Care Financing Review 1982;3(3):89-109.

31. Manton KG, Stallard E. Recent trends in mortality analysis. Orlando, FL: Academic Press; 1984.

32. Manton KG, Stallard E, Burdick D, Tolley HD. A stochastic compartment model of stomach cancer with correlated waiting time distributions. International Journal of Epidemiology 1979;8(3):283-91.

33. Manton KG, Stallard E, Vaupel JW. Alternative models for the heterogeneity of mortality risks among the aged. Journal of the American Statistical Association 1986;81(395):63544. 
34. Miller DG. On the nature of susceptibility to cancer. Cancer 1980;46:1307-18.

35. Moolgavkar SH. Carcinogenesis modeling: from molecular biology to epidemiology. Annual Review of Public Health 1986;7:151-69.

36. Mueller H, Weber W, editors. Familial cancer. Proceedings of the $1^{\text {st }}$ International Research Conference on Familial Cancer; 1985 Sep 16-21; Basel, Schweiz. Basel: Karger; 1985.

37. Muir CS. Epidemiological identification of cancer hazards. In: Nieburgs HE, editor. Prevention and detection of cancer. Part 2; Detection; vol 1: high risk markers, detection methods and management. Proceedings of the Third International Symposium on Detection and Prevention of Cancer; 1976 Apr 26 to May 1; New York. New York: Dekker; 1978. p. 53.

38. Muir CS, Nectoux J. International patterns of cancer. In: Schottenfeld D, Fraumeni JF Jr, editors. Cancer epidemiology and prevention. Philadelphia: Saunders; 1982. p. 119-37.

39. Newell GR. Epidemiology of Cancer. In: DeVita VT Jr, Hellman S, Rosenberg SA, editors. Cancer: principles and practice of oncology. $2^{\text {nd }}$ ed. Philadelphia: Lippincott; 1985. p. 151.

40. Nicholson WJ. Selection factors in cohort studies. National Cancer Institute Monograph 1985;67:111-5.

41. Nieburgs HE, editor. Prevention and detection of cancer. Part 2; Detection; vol 1: high risk markers, detection methodes and management. Proceedings of the Third International Symposium on Detection and Prevention of Cancer; 1976 Apr 26 to May 1; New York. New York: Dekker; 1978.

42. Nordling CO. A new theory on the cancer-inducing mechanism. British Journal of Cancer 1953;7:68-72.

43. Ooi WL, Elston RC, Chen VW, Bailey-Wilson JE, Rothschild H. Increased familial risk for lung cancer. Journal of the National Cancer Institute 1986;76(2):217-222.

44. Peto R. Epidemiology, multistage models, and short-term mutagenicity tests. In: Hiatt HH, Watson JD, Winsten JA, editors. Origins of human cancer. Book C: Human risk assessment. Proceedings of a conference; 1976 Sep. Cold Spring Harbor, NY: Cold Spring Harbor Laboratory; 1977. p. 1403.

45. Peto J. Genetic predisposition to cancer. In: Cairns J, Lyon JL, Skolnick M, editors. Cancer incidence in defined populations. Banbury Report; 4. Cold Spring Harbor, NY: Cold Spring Harbor Laboratory; 1980. p. 203.

46. Preston SH, Keyfitz N, Schoen R. Causes of death: life tables for national populations. New York: Seminar Press; 1972.

47. Sandstad B. Prediction of cancer incidence based on cohort analysis. In: Magnus K, editor. Trends in cancer incidence: causes and practical implications. Proceedings of a symposium; 1980 Aug 6-7; Oslo, Norway. Washington: Hemisphere Publishing; 1982.

48. Schoenberg BS. Multiple primary malignant neoplasms: the Connecticut experience, 1935-1964. Recent results in cancer research; 58. Berlin; New York: Springer; 1977.

49. Schottenfeld D, Fraumeni JF Jr, editors. Cancer epidemiology and prevention. Philadelphia: Saunders; 1982. 
50. Segi M, Tominaga S, Aoki K, Fujimoto I, editors. Cancer mortality and morbidity statistics: Japan and the world. GANN monograph on cancer research; 26. Tokyo: Japan Scientific Societies Press; 1981.

51. Stern AC, editor. The effect of air pollution. Air pollution; 2: Environmental Sciences. New York: Academic Press; 1977.

52. Teppo L. Incidence trends of melanoma of the skin in Finland by histological type. In: Magnus K, editor. Trends in cancer incidence: causes and practical implications.

Proceedings of a symposium; 1980 Aug 6-7; Oslo, Norway. Washington: Hemisphere Publishing; 1982. p. 393.

53. Tuyns AJ. Incidence trends of laryngeal cancer in relation to national alcohol and tobacco consumption. In: Magnus K, editor. Trends in cancer incidence: causes and practical implications. Proceedings of a symposium; 1980 Aug 6-7; Oslo, Norway. Washington: Hemisphere Publishing; 1982. p. 199.

54. Vaupel JW, Manton KG, Stallard E. The impact of heterogeneity in individual frailty on the dynamics of mortality. Demography 1979;16(3):439-54.

55. Vaupel JW, Yashin AI. The deviant dynamics of death in heterogeneous populations. Sociological Methodology 1985;15:179-211.

56. Vaupel JW, Yashin AI. Heterogeneity's ruses: some surprising effects of selection on population dynamics. American Statistician 1985;39(3):176-85.

57. Vaupel JW, Yashin AI, Manton KG. Debilitation's aftermath: stochastic process models of mortality. Mathematical Population Studies 1986;1(1):21-48.

58. Waterhouse $\mathrm{J}$ et al, editors. Cancer incidence in five continents: a technical report. Vol 3. IARC scientific publications, 0300-5085; 42. Lyon: International Agency for Research on Cancer; 1976.

59. Watson G. Age incidence curves for cancer. Proceedings of the National Academy of Science 1977;74(4):1341-2.

60. Yashin AI, Manton KG, Vaupel JW. Mortality and aging in a heterogeneous population: a stochastic process model with observed and unobserved variables. Theoretical Population Biology 1985;27(2):157-75.

61. Yashin AI, Manton KG, Stallard E. Dependent competing risks: a stochastic process model. Journal of Mathematical Biology 1986;24(2):119-140. 